\title{
Prioritizing connection requests in GMPLS-controlled optical networks
}

\author{
Ruepp, Sarah Renée; Koster, A.; Andriolli, N.; Manolova, Anna Vasileva
}

Published in:

International Conference on Photonics in Switching, 2009. PS '09

Link to article, DOI:

10.1109/PS.2009.5307736

Publication date:

2009

Document Version

Publisher's PDF, also known as Version of record

Link back to DTU Orbit

Citation (APA):

Ruepp, S. R., Koster, A., Andriolli, N., \& Manolova, A. V. (2009). Prioritizing connection requests in GMPLScontrolled optical networks. In International Conference on Photonics in Switching, 2009. PS '09 (pp. 1-1). IEEE. https://doi.org/10.1109/PS.2009.5307736

\section{General rights}

Copyright and moral rights for the publications made accessible in the public portal are retained by the authors and/or other copyright owners and it is a condition of accessing publications that users recognise and abide by the legal requirements associated with these rights.

- Users may download and print one copy of any publication from the public portal for the purpose of private study or research.

- You may not further distribute the material or use it for any profit-making activity or commercial gain

- You may freely distribute the URL identifying the publication in the public portal 


\title{
Prioritizing Connection Requests in GMPLS-Controlled Optical Networks
}

\author{
S. Ruepp ${ }^{1}$, A. Koster ${ }^{2}$, N. Andriolli ${ }^{3}$, A.V. Manolova ${ }^{1}$ \\ 1: Network Technologies and Service Platforms, DTU Fotonik, Technical University of Denmark, Kgs. Lyngby, Denmark \\ 2: Lehrstuhl II für Mathematik, RWTH Aachen, Germany \\ 3: Scuola Superiore Sant'Anna, Pisa, Italy
}

\begin{abstract}
We prioritize bidirectional connection requests by combining dynamic connection provisioning with off-line optimization. Results show that the proposed approach decreases wavelength-converter usage, thereby allowing operators to reduce blocking-probably under bulk connection assignment or network reconfiguration.
\end{abstract}

Keywords: Optical networks, GMPLS, simulation

\section{Introduction}

The Wavelength Division Multiplexing (WDM) technology has shown to be the primary solution for fulfilling the ever increasing demand for capacity in optical transport networks. To setup a connection in such a network a route and a wavelength must be identified for each connection request. This process is referred to as the routing and wavelength assignment (RWA) problem. If possible, connections are allocated on continuous wavelength paths (wavelength continuity constraint). This is due to the fact that even though emerging technologies allow for the conversion between wavelengths [1], these devices are still very expensive and therefore only a limited number of wavelength converters (WCs) is likely to be introduced in the networks. Previous studies have shown that economic use of these WCs significantly decreases the blocking probability during the connection provisioning phase [2-4].

If all connection demands are known beforehand, the RWA problem can be solved off-line, allowing for the globally most resource efficient assignment of routes and wavelengths. However, in real-life networks traffic demands most often arrive dynamically, which obstructs the aforementioned global optimization, since decisions on connection allocation must be made on the fly without knowledge of other ongoing and future connection demands. There are however two situations in particular, where such knowledge is available so that the off-line and the dynamic RWA can be favorably combined: first, if a set of connections have to be admitted to the network all at once as a bulk; and second, if reconfiguration of the network is carried out [5]. In this paper, we therefore show how dynamic and off-line RWA can be combined to save critical WC resources.

\section{Prioritizing bidirectional connections}

In optical core networks, connections are generally bidirectional and for ease of control, it is desirable that both directions are routed along the same links and use the same wavelength in both directions [6]. The two following subsections detail the proposed off-line prioritization and dynamic bidirectional connection provisioning approach.

\subsection{Off-line prioritization of requests}

The off-line optimization tool proposed in [3] returns an ordering of the connection requests, so that instead of the requests being treated based on a memory-less distribution, the same set of requests is reordered so that all requests using the same wavelength in the off-line solution are grouped. Demands that need conversion along the path are prioritized according to the wavelength used. Alternatively, we prioritize the demands according to the hop count measure. Due to this prioritization, the sequential dynamic setup of the connections is influenced to assign the routes and wavelengths of the off-line solution.

\subsection{Dynamic bidirectional connection setup}

Dynamic connection setup is carried out by using the RSVP-TE protocol. A flag within the Path message indicates that the connection request is bidirectional [7]. In order to minimize the usage of expensive resources, e.g., WCs, the Label Set [8] has been enhanced with the Suggested Vector [2], which is an additional object passed during connection setup that can be used to minimize the use of WCs. The concept is illustrated in figure 1. At each intermediate node, the Label Set is updated to reflect which wavelengths are available in both directions on a given link, while the Suggested Vector reveals the number of necessary wavelength conversions the choice of a given wavelength entails. When the connection request arrives at the destination node, it selects the wavelength requiring fewest WCs within the received Label Set (i.e., $\lambda_{2}$ in the example) and initiates the backward reservation of both connection directions on the chosen wavelength by passing the Resv message.

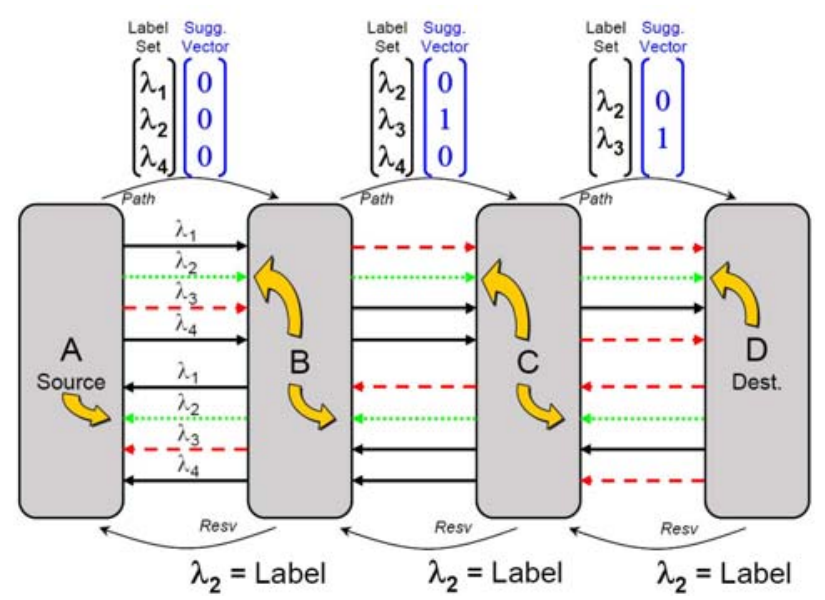

Figure 1: Bidirectional Connection Setup following [6] enhanced with Suggested Vector for WC minimization. 


\section{Simulation scenario and results}

We are using OPNET Modeler [9], a commercial discrete event simulation tool, combined with CPLEX-based off-line optimization to simulate connection allocation in a GMPLS-controlled network. As a test instance, we are using the Pan-European topology [10] illustrated in figure 2. The network consists of 28 nodes and 61 links, each equipped with one fiber of 10 wavelengths per direction.

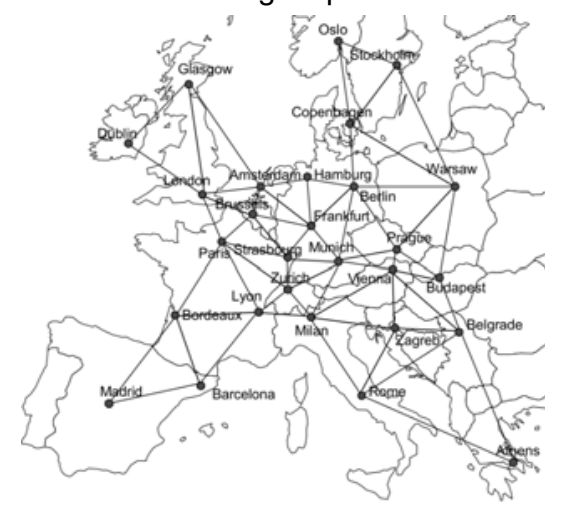

Figure 2: Pan-European triangular topology network [10].

In the dynamic scenario we only use Suggested Vector based dynamic wavelength assignment, i.e., no prioritization scheme is implemented. In the wavelength prioritized scenario, the optimization tool prioritizes requests that require WCs according to the highest wavelength used. In the hop count prioritized scenario, requests are prioritized according to descending hop counts.

The results illustrated in figure 3 show that the two proposed prioritization schemes decrease the WC usage, and hence avoid WC-bottleneck situations causing connection blocking. At low network loads, prioritizing the requests according to their assigned wavelength returned by the off-line optimization gives the best performance, while prioritizing requests in relation to decreasing hop count is more advantageous for high network loads. This is likely due to the increased number of conversions needed in the off-line optimized sequence as well.

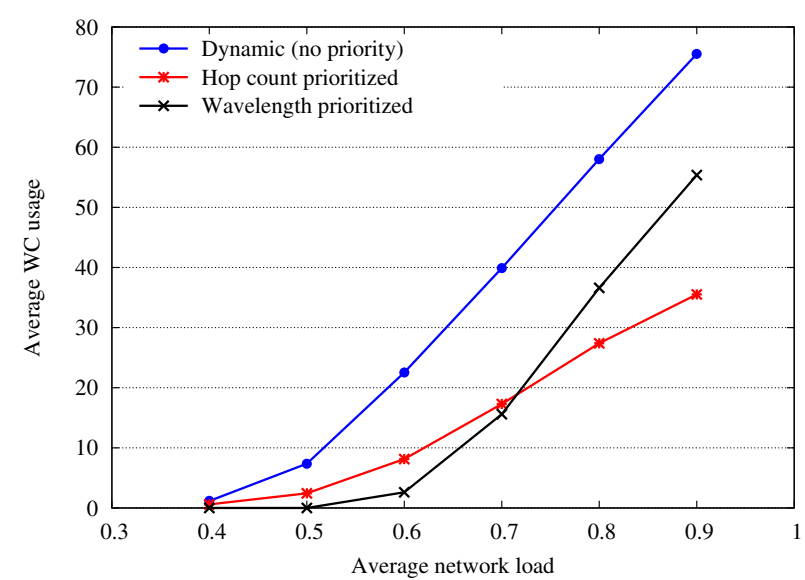

Figure 3: Average wavelength converter usage for dynamic wavelength assignment compared to when requests are prioritized according to wavelength usage or hop count.

\section{Conclusion}

In this paper, we investigate the performance of prioritizing bidirectional connection requests aiming at decreasing the use of WCs, which have shown to be the bottleneck for successful connection provisioning. We combine dynamic connection accommodation with off-line optimization and show that the WC usage can be significantly decreased compared to entirely dynamic wavelength assignment.

Our simulation results show that for low network loads, WC minimization can be achieved by prioritizing the demands according to the wavelength returned by the off-line optimization, while prioritizing the demands according to hop count is most beneficial under high network load conditions. These results illustrate that off-line RWA can be favorably combined with dynamic connection provisioning, which network operators can benefit from in case of desired bulk connection allocation or network reconfiguration.

\section{Acknowledgment}

The work described in this paper was carried out with the support of Cost Action 293, and the BONE-project ("Building the Future Optical Network in Europe"), a Network of Excellence funded by the European Commission through the 7th ICT-Framework Programme.

\section{References}

[1] B. Ramamurthy and B. Mukherjee, "Wavelength conversion in WDM networking," IEEE J. Sel. Areas Commun., vol. 16, no. 7, pp. 1061-1073, 1998.

[2] N. Andriolli et al. "Label Preference Schemes in GMPLS Controlled Networks", IEEE Communication Letters, vol. 10, no. 12, pp. 849-851, Dec. 2006

[3] A. Koster and S. Ruepp, "Benchmarking RWA Strategies for Dynamically Controlled Optical Networks", in Proc. of NETWORKS 2008

[4] S. Ruepp et al. "Restoration in All-Optical GMPLS Networks with Limited Wavelength Conversion", Computer Networks, vol. 52, no. 10, pp. 1951-1964, July 2008

[5] Roshani et al. "Reconfigurable Optical Networks: Is it Worth?", OTuA2, OFC 2008

[6] G. Bernstein et al. "Signaling Extensions for Wavelength Switched Optical Networks", draft-bernstein-ccamp-wsonsignaling-03.txt, Oct. 2008, Internet Draft, exp. Apr. 2009

[7] A. Farrel et al. "Encoding of Attributes for MPLS LSP Establishment Using Resource Reservation Protocol Traffic Engineering (RSVP-TE)", RFC 5420, Feb. 2009

[8] L. Berger (ed.), "Generalized Multi-Protocol Label Switching (GMPLS) Signaling Functional Description," IETF RFC 3471, Jan. 2003.

[9] OPNET Technologies, Inc. www.opnet.com

[10] Inkret, R., Kuchar, A. and Mikac, B. (eds.), "Extended Final Report of COST Action 266 - Advanced Infrastructure for Photonic Networks", 2003 